\title{
Breaking the Contrast Limit in Single-Pass Fabry-Pérot Spectrometers
}

\author{
G. Antonacci, ${ }^{1, *}$ S. De Panfilis, ${ }^{1}$ G. Di Domenico, ${ }^{1,2}$ E. DelRe, ${ }^{2,3}$ and G. Ruocco ${ }^{1}$ \\ ${ }^{1}$ Center for Life Nano Science@Sapienza, Istituto Italiano di Tecnologia, 00161 Rome, Italy \\ ${ }^{2}$ Dipartimento di Fisica, Università di Roma "La Sapienza", 00185 Rome, Italy \\ ${ }^{3}$ CNR-ISC, Università di Roma "La Sapienza", 00185 Rome, Italy \\ (Received 1 September 2016; revised manuscript received 21 October 2016; published 29 November 2016)

\begin{abstract}
The development of high-resolution Fabry-Pérot interferometers has enabled a wide range of scientific and technological advances-ranging from the characterization of material properties to the more fundamental studies of quasiparticles in condensed matter. Spectral contrast is key to measuring weak signals and can reach a $10^{3}$ peak-to-background ratio in a single-pass assembly. At its heart, this limit is a consequence of an unbalanced field amplitude across multiple interfering paths, with an ensuing reduced fringe visibility. Using a high-resolution, high-throughput virtually imaged phased array spectrometer, we demonstrate an intensity-equalization method to achieve an unprecedented 1000-fold increase in spectral contrast in a single-stage, single-pass configuration. To validate the system, we obtain the Brillouin spectrum of water at high scattering concentrations the interferometer close to its ultimate limits and allows rapid high-resolution spectral analysis in a wide range of fields, including Brillouin spectroscopy, mechanical imaging, and molecular fingerprinting.
\end{abstract} \\ where, unlike with the standard scheme, the inelastic peaks are highly resolved. Our method brings
}

DOI: 10.1103/PhysRevApplied.6.054020

\section{INTRODUCTION}

Fabry-Pérot (FP) interferometers have been extensively used in optical spectroscopy to measure small spectral variations with a sub-GHz $\left(<0.03 \mathrm{~cm}^{-1}\right)$ resolution, in turn enabling biological and chemical sensing, material analysis, and light-source characterization. For example, Brillouin light-scattering spectroscopy uses FP interferometers and etalons to measure mechanical properties of materials such as stiffness [1,2], phase transitions [3], relaxation dynamics [4], and elastic constants $[5,6]$.

The limit on the spectral contrast achievable by standard FP interferometers and etalons is ultimately dictated by the transmission Airy function describing the intensity profile of the far-field interference pattern. Specifically, the Airy function results from the Fourier transform of the output exponentially decaying field [Fig. 1(a)], which gives rise to Lorentzian peaks characterized by long frequency tails, decaying as $\nu^{-2}$, that limit the contrast [7,8] [Fig. 1(b)]. At its origin, this limit arises from an unbalanced field amplitude of the multiple interfering beams, with an ensuing reduced fringe visibility.

We present a method for greatly increasing the spectral contrast of a Fabry-Pérot spectrometer by equalizing the interfering transmitted field components. Using a Gaussian equalization, we achieve a 1000-fold increase in spectral

*giuseppe.antonacci@iit.it contrast in a commercial high-transmission single-stage, single-pass spectrometer.

Our equalization technique is demonstrated on a virtually imaged phased array (VIPA), a parallel FP etalon where the input beam is focused to an antireflection (AR) dielectric-coated window that minimizes insertion losses [9]. Although standard multipass FP spectrometers typically provide higher contrast and resolution, the VIPA is especially important because it manifests a superior $(>50 \%)$ throughput efficiency and performs a scan-free detection of the light spectrum on a single CCD frame. Given their rapid $(<1 \mathrm{~s})$ spectral acquisition, VIPA etalons have enabled the extension of Brillouin spectroscopy into a promising imaging technique for noninvasively assessing the biomechanics in the volume of biological systems [10-12]. Moreover, VIPA etalons have been used in molecular fingerprinting to separate the stabilized frequency comb of a femtosecond laser [13]. As in a standard FP interferometer, the multiple internal reflections within the VIPA cavity yield the phase conditions for interferometrically separating the input spectral components.

\section{METHODS}

\section{A. Transmission functions}

Figure 1(c) plots the etalon Airy function compared with a transmission function given by an equalized intensity profile. The sum of the $N$ exponentially decaying fields transmitted by an etalon [Fig. 1(a)] gives the total amplitude: 
(a)

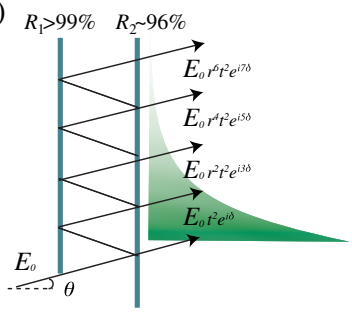

(c)
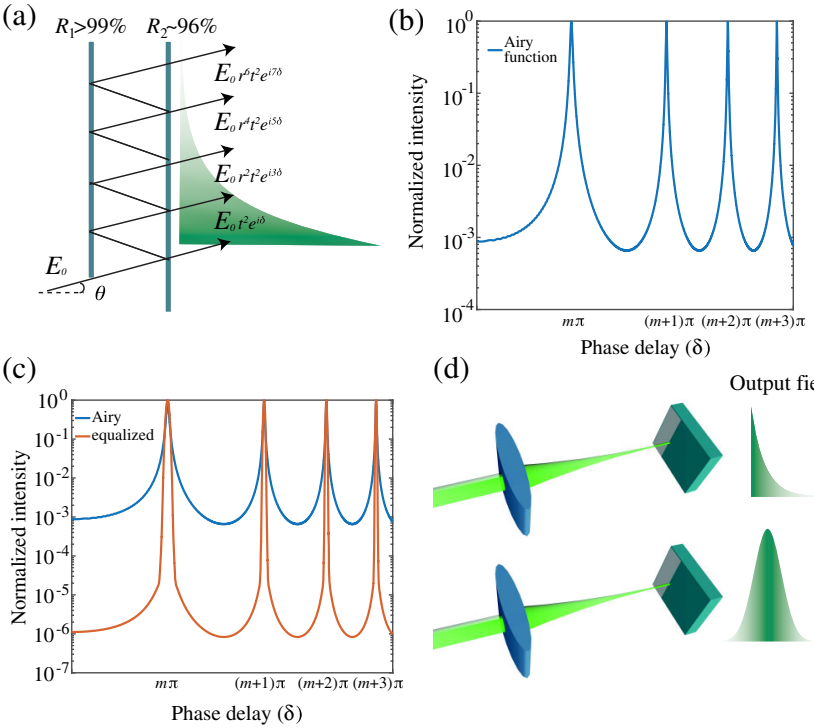

(d)

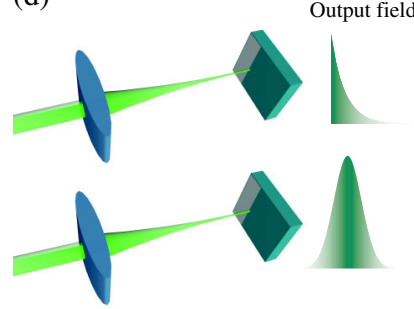

(e)

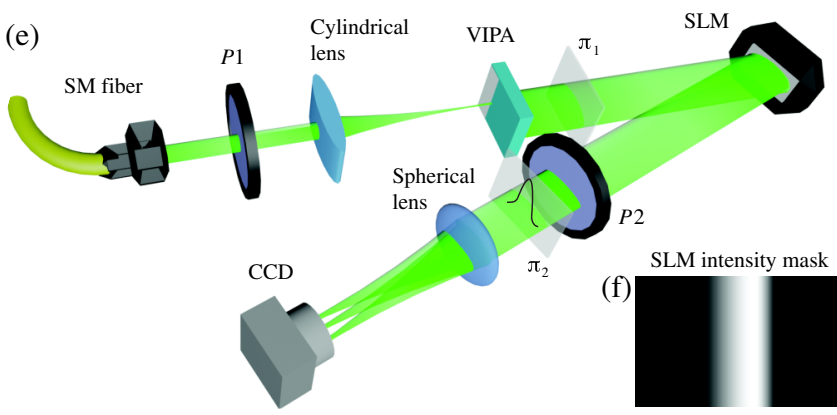

FIG. 1. (a) Schematic of the transmitted field of a VIPA etalon. The incident beam is internally reflected by a high- $\left(R_{1} \sim 99.9 \%\right)$ and a low- $\left(R_{2} \sim 96 \%\right)$ reflection coated surface. (b) The Airy function given by the output exponentially decaying field ultimately sets a limit (of approximately $10^{3}$ ) to the instrumental spectral contrast. (c) A 1000-fold increase in contrast is obtained when we assume an output field amplitude (d) of Gaussian profile. (e) Schematic of the spectrometer $\left(P_{i}\right.$, linear polarizer; $\pi_{i}$, field observation plane). The light beam delivered by a singlemode (SM) fiber is focused by a cylindrical lens to the AR-coated window of a VIPA etalon, giving high $(>50 \%)$ throughput efficiency. A SLM is used to equalize the output field of the VIPA through an intensity mask of semi-Gaussian profile (f). A CCD camera acquires the spectral pattern at the Fourier plane of a spherical lens.

$$
\begin{aligned}
E_{t} & =E_{0}\left[t^{2} e^{i \delta}+r^{2} t^{2} e^{i 3 \delta}+r^{4} t^{2} e^{i 5 \delta}+\cdots\right] \\
& =E_{0} T e^{i \delta} \sum_{j=0}^{N} R^{j} e^{i 2 \delta j} \\
& =E_{0} \frac{T e^{i \delta}}{1-R e^{2 i \delta}},
\end{aligned}
$$

where $R=|r|^{2}$ and $T=|t|^{2}$ are the reflection and transmission intensity coefficients, respectively, and $\delta$ is the phase delay of the incident beam in a single-cavity pass. As a result, the total transmitted intensity distribution is given by

$$
\frac{I_{t}}{I_{0}}=\left|\frac{E_{t}}{E_{0}}\right|^{2}=\frac{1}{1+F \sin ^{2} \delta},
$$

with

$$
F=\frac{4 R}{(1-R)^{2}}
$$

Equation (2) is the well-known Airy function describing the intensity profile of the total transmitted field of a Fabry-Pérot interferometer or etalon. In turn, our equalized transmission function $I_{\mathrm{eq}}$ arises from fields forming a Gaussian amplitude profile at the output [Fig. 1(d)] and sharing the same phase pattern of the standard case,

$$
\frac{I_{\mathrm{eq}}}{I_{0}}=\left|T e^{i \delta} \sum_{j=0}^{N} e^{-\frac{\left(j-j_{0}\right)^{2}}{2 \sigma^{2}}} e^{i 2 \delta j}\right|^{2},
$$

where $j_{0}$ and $\sigma$ are the center and the width of the Gaussian profile [Fig. 1(c)]. Given the much faster decay of the tails of a Gaussian function than those quadratics present in a Lorentzian function, our equalized transmission profile manifests up to a 1000-fold increase in the peak-to-background ratio with respect to the standard Airy function.

\section{B. Experimental details}

The high flatness and parallelism required across the surfaces of the VIPA cavity impose the application of a homogeneous reflection coating on both etalon surfaces. An ideal Gaussian reflectivity profile at the output surface of the VIPA etalon would, indeed, involve the application of several dielectric coating layers varying the effective cavity-optical path lengths - and thus the phase pattern of the output field-which, in turn, would lead to a complex, nonperiodic interference pattern. An attempt to apodize VIPA spectrometers was made using linear-variable neutral-density filters [14]. However, despite a smoothed beam shape, the improper linear transfer function results in an unbalanced intensity profile with an ensuing limited contrast gain. To overcome these limitations, we integrate a spatial light modulator (SLM) to shape the output beam of the VIPA [Fig. 1(e)] by displaying an appropriate intensity mask of semi-Gaussian profile [Fig. 1(f)] along the dispersion axis of the etalon.

The light beam of a single-longitudinal-mode laser (Coherent Verdi, $\lambda=532 \mathrm{~nm}$ ) is delivered by a standard single-mode (SM) fiber (Thorlabs, p3-460b) and focused by a cylindrical lens $(f=200 \mathrm{~mm})$ to a line at the ARcoated window of the VIPA etalon (LightMachinery), which had a free spectral range (FSR) of $30 \mathrm{GHz}$ $\left(1 \mathrm{~cm}^{-1}\right)$. The SLM (Holoeye Pluto) is set in amplitude mode through an appropriate choice of the input $\left(P_{1}\right)$ and output $\left(P_{2}\right)$ polarizers. To find the optimal Gaussian profile of the modulated beam, the SLM intensity mask is adjusted 

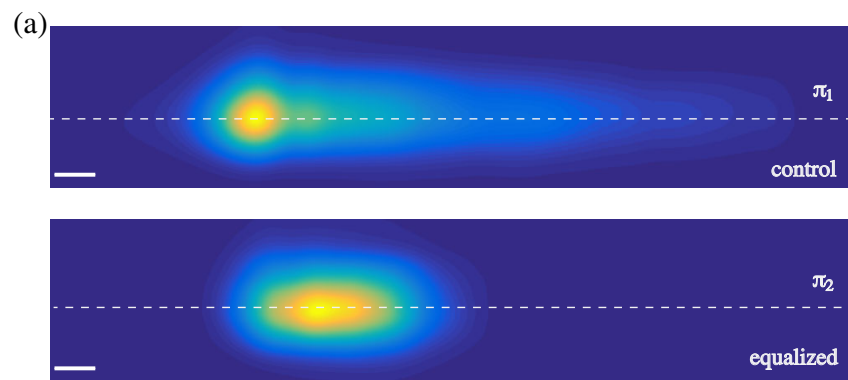

(b)

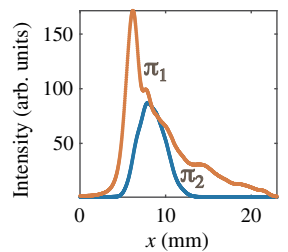

(c)

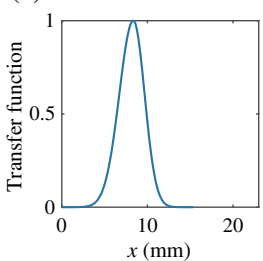

(d)

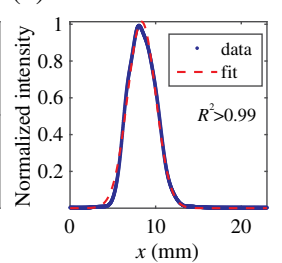

FIG. 2. Beam shaping for high spectral contrast. (a) Comparison of the output beam of the VIPA acquired at the $\pi_{1}$ plane with the SLM-equalized beam at the $\pi_{2}$ plane. (b) Line profiles (the dashed lines) along the two beams acquired with an equal integration time. Using a transfer function of semi-Gaussian profile (c), the natural exponentially decaying beam arising at the VIPA output is converted into a Gaussian beam of an approximately $5 \mathrm{~mm}$ width. (d) A real-time least-squares fit is performed to achieve the optimal beam shape $\left(R^{2}>0.99\right)$ with a loss of approximately $58 \%$. Scale bars are in $2-\mathrm{mm}$ increments.

according to the minimal uncertainty given by a realtime least-squares fitting of the beam. The resulting Gaussian beam is Fourier transformed by a spherical lens $(f=200 \mathrm{~mm})$ so as to separate the interference orders and to yield the final spectral pattern, which is acquired from a multichannel CCD camera (Photometrix Prime) in a single frame.

Figure 2(a) compares the natural field of the VIPA acquired at the output plane $\left(\pi_{1}\right)$ with the equalized field given by the SLM (the $\pi_{2}$ plane). An integrated line profile of the two fields with their relative intensities is illustrated in Fig. 2(b). The output field of the etalon shows an exponentially decaying profile as a result of the multiple internal reflections occurring inside the etalon cavity. In turn, the equalized field given by the SLM shows a Gaussian profile. The appropriate transfer function [Fig. 2(c)] is applied to the SLM intensity mask during the modulation process, giving a Gaussian fit of $R^{2}>0.99$ [Fig. 2(d)] and an integrated intensity loss of approximately $58 \%$. This loss may be strongly reduced by using an alternative phase mask with the SLM operating in phase rather than in amplitude mode, but a change in the phase distribution of the output beam would also affect the resulting interference pattern. Considering the additional losses (typically of approximately 40\%) arising from the VIPA etalon, we estimate the overall system-throughput efficiency to be more than $25 \%$.

\section{RESULTS}

Figure 3 shows the spectral contrast measured with both a standard and an equalized single-stage VIPA configuration. To overcome the limited dynamic range of the CCD camera, we use a set of calibrated neutral-density (ND) filters and reconstruct the spectral profiles according to the filter attenuation levels. The spectral contrast given by the single-stage VIPA spectrometer is measured to be approximately $32 \mathrm{~dB}$ at half FSR, in line with previous reports [15]. In turn, the contrast obtained through the Gaussian equalization is measured to be approximately $60 \mathrm{~dB}$, corresponding to a more-than-25-dB gain with respect to the standard setup and in agreement with our theoretical predictions. The spectral resolution of both setups is measured to be about $800 \mathrm{MHz}$, corresponding to a finesse of approximately 40 and a resolving power of approximately $8 \times 10^{5}$.

The enhanced contrast of our spectrometer is demonstrated in a Brillouin light-scattering scheme, where both high spectral resolution and high contrast are key to measuring the weak-inelastic Brillouin peaks. Brillouin spectra of a water-milk solution are acquired in a fraction of a second at different milk concentrations with both the standard [Fig. 4(a)] and the intensity-equalized [Fig. 4(b)] single-stage VIPA spectrometer. The spectrum given by distilled water is typically characterized at room temperature by a side Stokes and an anti-Stokes Brillouin peak, and a central Rayleigh peak of lower intensity. This spectral profile is clearly observed in both configurations, where the Brillouin shift is measured to be $\nu_{B}=5.05 \pm 0.03 \mathrm{GHz}$, in agreement with previous reports [16]. As the scattering milk concentration increases [Fig. 4(c)], the Rayleigh peak

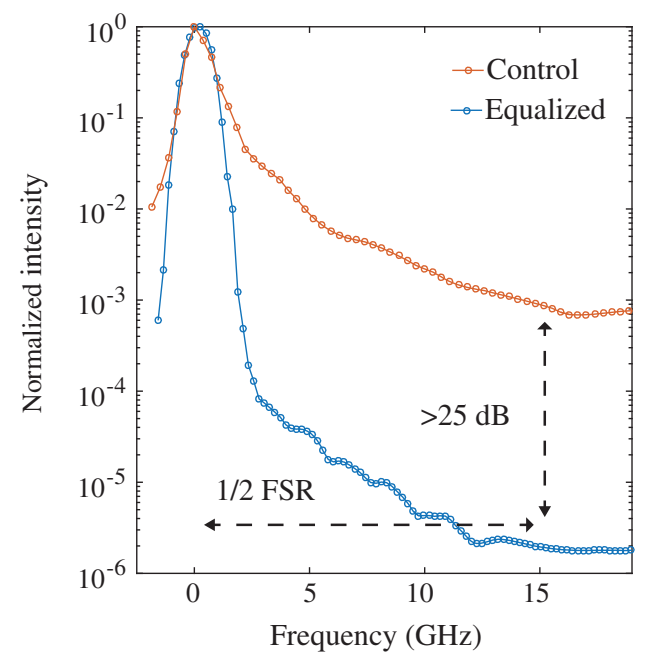

FIG. 3. Spectral contrast. The spectral profile is reconstructed using a set of calibrated ND filters. The contrast given by our spectrometer is measured to be approximately $60 \mathrm{~dB}$ at half FSR, corresponding to a more-than-25-dB increase with respect to the contrast given by a standard single-stage VIPA spectrometer. 

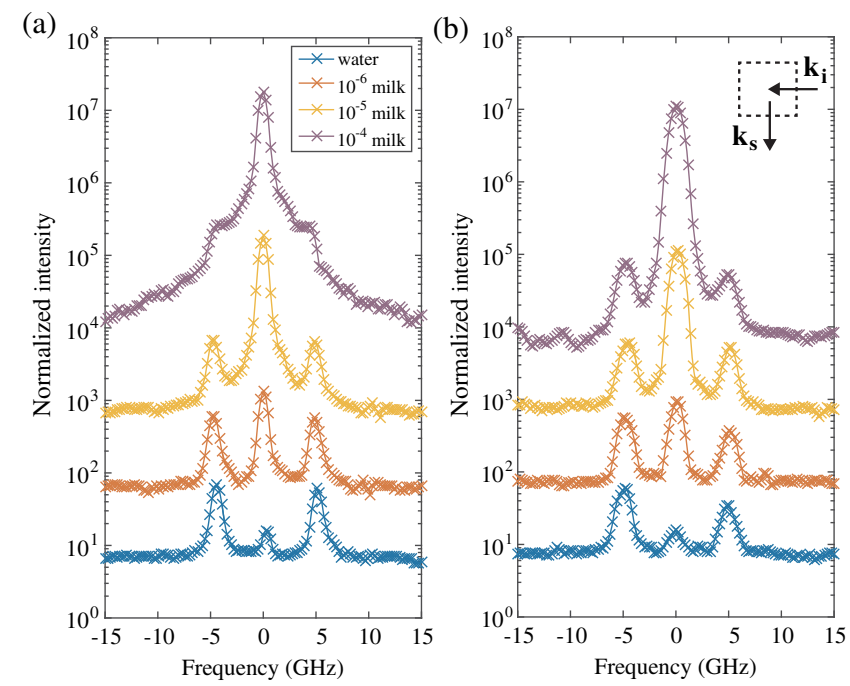

(c)

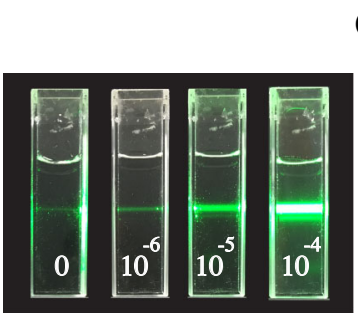

(d)

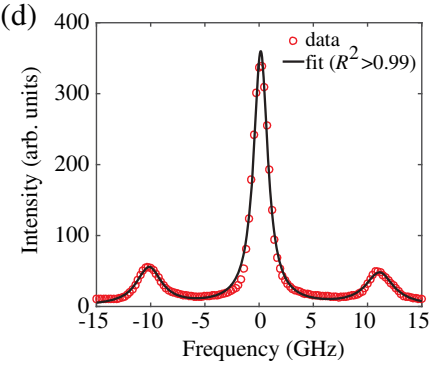

FIG. 4. Validation of the spectrometer. Brillouin spectra of a water-milk solution at different milk concentrations acquired in a $90^{\circ}$ scattering geometry with both (a) the standard and (b) the equalized single-stage VIPA spectrometer. An arbitrary offset is added to the spectral profiles at each milk concentration for clarity. (c) Scattering of the green laser beam through the liquid samples. The spectrum of distilled water at room temperature has a lower Rayleigh peak than the inelastic Brillouin peaks, as shown in both configurations. As the milk concentration increases, elastic scattering prevails, overwhelming the Brillouin peaks (a). In turn, Brillouin peaks are still highly resolved at $10^{-4}$ milk concentration due to the higher contrast of the spectrometer (b). Spectra are acquired with a 200-ms integration time. (d) Brillouin spectrum of glycerol. Glycerol is characterized by a natural high Rayleigh peak and much weaker Brillouin peaks. $\mathbf{k}_{\mathbf{i}}$ and $\mathbf{k}_{\mathbf{s}}$ represent incident and scattering wave vectors.

associated with the elastic component of the spectrum exhibits a sharp growth. At $10^{-4}$ milk concentration, the Brillouin peaks cannot be measured by the standard singlestage VIPA spectrometer, where the elastic background completely overwhelms the spectral lines due to a lack of contrast [Fig. 4(a)]. Conversely, the Brillouin peaks are still highly resolved as the intensity equalization is applied, demonstrating the higher contrast of our system [Fig. 4(b)].

As a further example, Fig. 4(d) shows a typical spectrum of glycerol characterized by a naturally stronger Rayleigh peak and substantially weaker Brillouin peaks than those arising from standard liquids, as dictated by the LandauPlaczek ratio [17]. Brillouin shift is measured to be
$\nu_{B}=10.62 \pm 0.04 \mathrm{GHz}$, in agreement with previous reports [18]. These findings validate our system as a robust high-contrast spectrometer.

\section{DISCUSSION AND CONCLUSION}

Spectral contrast is a key ingredient for the detection of weak spectral components arising from light-matter processes. For example, inelastic spectral lines associated with shear and surface waves of solid-state materials, such as glasses, are typically several orders of magnitude weaker than the elastic Rayleigh peak and cannot be detected by a single FP interferometer due to the poor contrast [19], thus leaving a stark lack of fundamental information. Many schemes, such as destructive interference [20], line absorption [21], and FP bandpass filtering [22], have been proposed to suppress the elastic component of the spectrum when strong Rayleigh scattering and specular reflections are collected by the system. Nevertheless, these approaches leave the spectral contrast unvaried. Current methods for increasing the spectral contrast involve the use of multiple spectral devices, such as FP etalons and diffraction gratings, placed in a series and operating in a beam multipassing mode [23-25]. For example, the gold-standard spectrometer of the Sandercock type is composed of a tandem of scanning FP interferometers working in a triplepass configuration, which can reach a peak-to-background ratio of up to $10^{15}$ and a finesse of 60 [26]. A similar approach is proposed using multistage VIPA etalons, where an approximately-55-dB spectral contrast is given by a crossed dispersion axis [15]. However, multistage spectrometers involve a large number of optical and mechanical components, which make them expensive, unstable, and low efficient.

The equalization technique discussed here hinges on a basic interferometric principle that can achieve high spectral contrast and a sub-GHz resolution without involving complex multistage architectures. Furthermore, involving an alternative path-equalization scheme, it can be independently combined with the other spectralcontrast-enhancement techniques developed in the last decades. Our method, therefore, can set a standard for rapid, highly efficient, low-cost spectral analysis in a wide range of topics, including Brillouin microscopy, atomic clocks, precision spectroscopy, and molecular fingerprinting.

\section{ACKNOWLEDGMENTS}

We acknowledge financial support from CrestOptics s.r.l.

[1] J. M. Vaughan and J.T. Randall, Brillouin scattering, density and elastic properties of the lens and cornea of the eye, Nature (London) 284, 489 (1980). 
[2] G. Antonacci, R. M. Pedrigi, A. Kondiboyina, V. V. Mehta, R. de Silva, C. Paterson, R. Krams, and P. Török, Quantification of plaque stiffness by Brillouin microscopy in experimental thin cap fibroatheroma, J. R. Soc. Interface 12, 20150843 (2015).

[3] M. Grimsditch, R. Bhadra, and Y. Meng, Brillouin scattering from amorphous materials at high pressures, Phys. Rev. B 38, 7836 (1988).

[4] C. J. Montrose, V. A. Solovyev, and T. A. Litovitz, Brillouin scattering and relaxation in liquids, J. Acoust. Soc. Am. 43, 117 (1968).

[5] K. J. Koski, P. Akhenblit, K. McKiernan, and J. L. Yarger, Non-invasive determination of the complete elastic moduli of spider silks, Nat. Mater. 12, 262 (2013).

[6] D. Schneider, N. Gomopoulos, C. Y. Koh, P. Papadopoulos, F. Kremer, E. L. Thomas, and G. Fytas, Nonlinear control of high-frequency phonons in spider silk, Nat. Mater. 15, 1079 (2016).

[7] O. Svelto, Principles of Lasers (Plenum, London, 1976).

[8] N. Ismail, C. Kores, D. Geskus, and M. Pollnau, Fabry-Pérot resonator: Spectral line shapes, generic and related Airy distributions, linewidths, finesses, and performance at low or frequency-dependent reflectivity, Opt. Express 24, 16366 (2016).

[9] M. Shirasaki, Large angular dispersion by a virtually imaged phased array and its application to a wavelength demultiplexer, Opt. Lett. 21, 366 (1996).

[10] G. Antonacci and S. Braakman, Biomechanics of subcellular structures by non-invasive Brillouin microscopy, Sci. Rep. 6, 37217 (2016).

[11] G. Scarcelli and S. H. Yun, Confocal Brillouin microscopy for three-dimensional mechanical imaging, Nat. Photonics 2, 39 (2008).

[12] G. Antonacci, Ph.D. thesis, Imperial College London, 2015.

[13] S. A. Diddams, L. Hollberg, and V. Mbele, Molecular fingerprinting with the resolved modes of a femtosecond laser frequency comb, Nature (London) 445, 627 (2007).

[14] G. Scarcelli, W. J. Polacheck, H. Y. Nia, K. Patel, A. J. Grodzinsky, R. D. Kamm, and S. H. Yun, Noncontact threedimensional mapping of intracellular hydromechanical properties by Brillouin microscopy, Nat. Methods 12, 1132 (2015).

[15] G. Scarcelli and S.H. Yun, Multistage VIPA etalons for high-extinction parallel Brillouin spectroscopy, Opt. Express 19, 10913 (2011).

[16] G. Antonacci, M. R. Foreman, C. Paterson, and P. Török, Spectral broadening in Brillouin imaging, Appl. Phys. Lett. 103, 221105 (2013).

[17] V. A. Popova and N. V. Surovtsev, Temperature dependence of the Landau-Placzek ratio in glass forming liquids, J. Chem. Phys. 135, 134510 (2011).

[18] L. Comez, D. Fioretto, F. Scarponi, and G. Monaco, Density fluctuations in the intermediate glass-former glycerol: A Brillouin light scattering study, J. Chem. Phys. 119, 6032 (2003),

[19] S. M. Lindsay, M.W Anderson, and J. R. Sandercock, Construction and alignment of a high performance multipass vernier tandem Fabry-Pérot interferometer, Rev. Sci. Instrum. 52, 1478 (1981).

[20] G. Antonacci, G. Lepert, C. Paterson, and P. Török, Elastic suppression in Brillouin imaging by destructive interference, Appl. Phys. Lett. 107, 061102 (2015).

[21] Z. Meng, A. J. Traverso, and V. V. Yakovlev, Background clean-up in Brillouin microspectroscopy of scattering medium, Opt. Express 22, 5410 (2014).

[22] A. Fiore, J. Zhang, P. Shao, S. H. Yun, and G. Scarcelli, High-extinction virtually imaged phased array-based Brillouin spectroscopy of turbid biological media, Appl. Phys. Lett. 108, 203701 (2016).

[23] J. G. Dil, N. C. Van Hijningen, F. Van Dorst, and R. M. Aarts, Tandem multipass Fabry-Pérot interferometer for Brillouin scattering, Appl. Opt. 20, 1374 (1981).

[24] S. M. Lindsay and I. W. Shepherd, A high-contrast multipass Fabry-Pérot spectrometer, J. Phys. E 10, 150 (1977).

[25] V. Mazzacurati, P. Benassi, and G. Ruocco, A new class of multiple dispersion grating spectrometers, J. Phys. E 21, 798 (1988).

[26] R. Mock, B. Hillebrands, and R. Sandercock, Construction and performance of a Brillouin scattering set-up using a triple-pass tandem Fabry-Pérot interferometer, J. Phys. E 20, 656 (1987). 